Proceedings

\title{
Reduced Graphene Oxide Fibre Electrodes for Drug Sensing †
}

\author{
Sutthima Sriprasertsuk, John R. Varcoe and Carol Crean *
}

Citation: Sriprasertsuk, S.; Varcoe, J.R.; Crean, C. Reduced Graphene Oxide Fibre Electrodes for Drug Sensing. Proceedings 2021, 68, 18. https://doi.org/10.3390/proceedings2021068018

\section{Published: 4 March 2021}

Publisher's Note: MDPI stays neutral with regard to jurisdictional claims in published maps and institutional affiliations.

\section{(c) (1)}

Copyright: (C) 2021 by the authors. Licensee MDPI, Basel, Switzerland. This article is an open access article distributed under the terms and conditions of the Creative Commons Attribution (CC BY) license (http://creativecommons.org/licenses /by/4.0/).
Department of Chemistry, University of Surrey, Guildford GU10 5PH, UK; s.sriprasertsuk@surrey.ac.uk (S.S.); J.Varcoe@surrey.ac.uk (J.R.V.)

* Correspondence: c.crean@surrey.ac.uk

† Presented at the International Conference on the Challenges, Opportunities, Innovations and Applications in Electronic Textiles (E-Textiles 2020), Virtual Venue, UK, 4 November 2020.

\begin{abstract}
Reduced graphene oxide (rGO) fibre electrodes and their ability to sense paracetamol (as model drug) were studied. rGO was electrodeposited onto carbon fibre by two different approaches: potentiostatic deposition and cyclic voltammetry $(\mathrm{CV})$ in the presence of graphene oxide solution. Carbon fibre electrodes coated with rGO (after five CV cycles) could sense paracetamol with an oxidation peak at $0.62 \mathrm{~V}$ (vs. $\mathrm{Ag} / \mathrm{AgCl}$ ). The limit of detection of this fibre sensor was found to be $36.3 \mu \mathrm{M}$ with a linear range of $50-500 \mu \mathrm{M}$ of paracetamol $\left(\mathrm{R}^{2}=0.9901\right)$.
\end{abstract}

Keywords: reduced graphene oxide; fibre electrode; cyclic voltammetry; drug sensing; wearable sensors

\section{Introduction}

Reduced graphene oxide (rGO) is widely studied as a potential electrode material for electrochemical sensors and biosensors due to its high electrical conductivity, large surface area, good biocompatibility and high electrocatalytic activity [1,2]. The oxygen-containing groups of GO are reduced leading to more $\mathrm{sp}^{2}$ hybridised carbon atoms with small amounts of hydroxyl $(-\mathrm{OH})$, carboxy $(-\mathrm{COOH})$, or epoxide $(-\mathrm{O}-)$ groups remaining $[3,4]$. To demonstrate the ability of rGO-coated fibre electrodes to sense drugs, the monitoring of model drug paracetamol was explored using cyclic voltammetry (CV). Additionally, the surface morphology and electrochemical surface areas of the fibre electrodes were analysed. Figure 1 illustrates the oxidation of paracetamol, an antipyretic drug, widely used to treat fever and relieve mild to moderate pain [5].

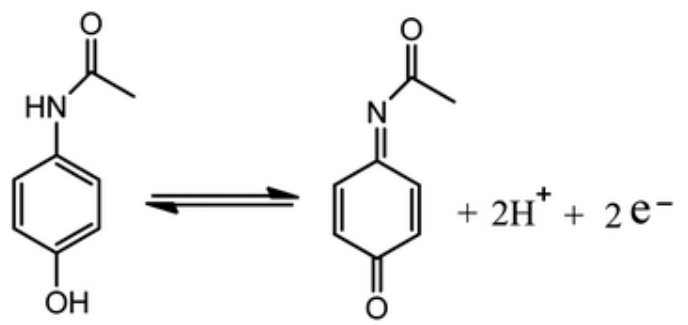

Figure 1. Schematic of the oxidation of paracetamol [5].

\section{Materials and Methods}

\subsection{Reagents}

Potassium ferricyanide $\left(\mathrm{K}_{3}\left[\mathrm{Fe}(\mathrm{CN})_{6}\right]\right)$, potassium chloride $(\mathrm{KCl})$, and paracetamol were also purchased from Sigma-Aldrich (UK). Phosphate buffered saline (PBS) tablets were purchased from Fisher Scientific (UK). Carbon fibre was purchased from Alfa Aesar (UK). 


\subsection{Apparatus}

All cyclic voltammetry (CV) was performed using an eDAQ EA161 potentiostat with eDAQ e-corder 401 supported by EChem V2.1.16 software. A Basi ${ }^{\circledast} \mathrm{Ag} / \mathrm{AgCl}$ in $3.0 \mathrm{M}$ $\mathrm{NaCl}$ was used as a reference electrode and a platinum mesh was used as a counter electrode. Raman spectroscopy was performed using a Renishaw inVia confocal Raman microscope with a $785 \mathrm{~nm}$ laser. Scanning electron microscopy (SEM) images were obtained using a JEOL USA JSM-7100 F field emission electron microscope.

\subsection{Procedure}

Graphene oxide ( $\left.0.5 \mathrm{mg} \mathrm{ml}^{-1}\right)$ was ultrasonically dissolved in $0.1 \mathrm{M}$ phosphate buffer solution. rGO was deposited onto carbon fibre using two different approaches: potentiostatic deposition and CV. For potentiostatic deposition, rGO was deposited by applying $-1.2 \mathrm{~V}(\mathrm{vs} . \mathrm{Ag} / \mathrm{AgCl}$ ) for $5 \mathrm{~min}$ onto carbon fibre. For deposition by $\mathrm{CV}$, a potential range of -1.4 to $+1.4 \mathrm{~V}$ (vs. $\mathrm{Ag} / \mathrm{AgCl}$ ) was used at a scan rate of $50 \mathrm{mV} \mathrm{s}^{-1}$; the number of cycles was varied from 3 to 5 to optimize the rGO sensing layer. The performance of the sensors with respect to paracetamol was tested. The three-electrode system was used for analysis by $\mathrm{CV}$ at a potential range of -0.6 to $+0.9 \mathrm{~V}$ (vs. $\mathrm{Ag} / \mathrm{AgCl}$ ) at a scan rate of $50 \mathrm{mV} \mathrm{s}^{-1}$ in the presence of paracetamol $(0-500 \mu \mathrm{M})$.

\section{Results and Discussion}

\subsection{Characterisation}

rGO was electrodeposited onto carbon fibre by potentiostatic deposition and cyclic voltammetry $(\mathrm{CV})$ in the presence of graphene oxide solution. Figure 2 shows the Raman spectrum of uncoated carbon fibre (black line) with peaks of weak intensity at 1330 and $1599 \mathrm{~cm}^{-1}$ corresponding to the D and G bands of carbonaceous material. The D band is attributed to $\mathrm{sp}^{3}$ hybridised carbon and disorder in the carbon lattice, while, the $\mathrm{G}$ band is assigned to the stretching of the $\mathrm{C}=\mathrm{C}$ bond [2]. Potentiostatic deposition of $\mathrm{rGO}$ (red line) and rGO deposited using three $\mathrm{CV}$ cycles onto carbon fibre (blue line) exhibits $\mathrm{D}$ and G band peaks at 1337 and $1592 \mathrm{~cm}^{-1}$, and 1325 and $1598 \mathrm{~cm}^{-1}$, respectively, that are shifted compared to those of the underlying carbon fiber.

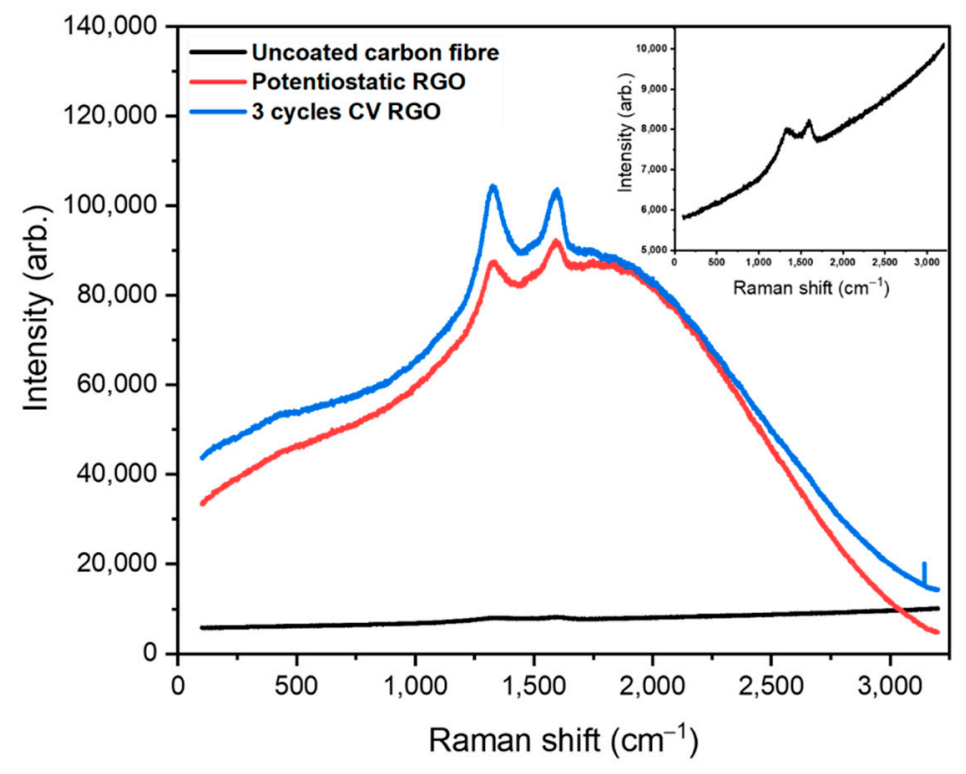

Figure 2. Raman spectrum (obtained using a $785 \mathrm{~nm}$ laser) of uncoated carbon fibre (black), carbon fibre coated with rGO by potentiostatic deposition (red) and carbon fibre coated with rGO using 3 CV cycles (blue). 
Scanning electron microscope images of uncoated carbon fibre (Figure 3a) shows a smooth surface, while carbon fibre coated with rGO using $3 \mathrm{CV}$ cycles (Figure $3 \mathrm{~b}$ ) displays a sponge-like morphology coating parts of the carbon fibre. Energy-dispersive $\mathrm{X}$-ray spectroscopy (EDS) results clearly show that the rGO coated on carbon fibre by three CV cycles was successful (Figure 3d), as the chemical composition of Carbon and Oxygen (table gives values for areas not coated (area 1) and coated with rGO (area 2)) confirms the existence of functionality consistent with rGO on the carbon fibre surface.
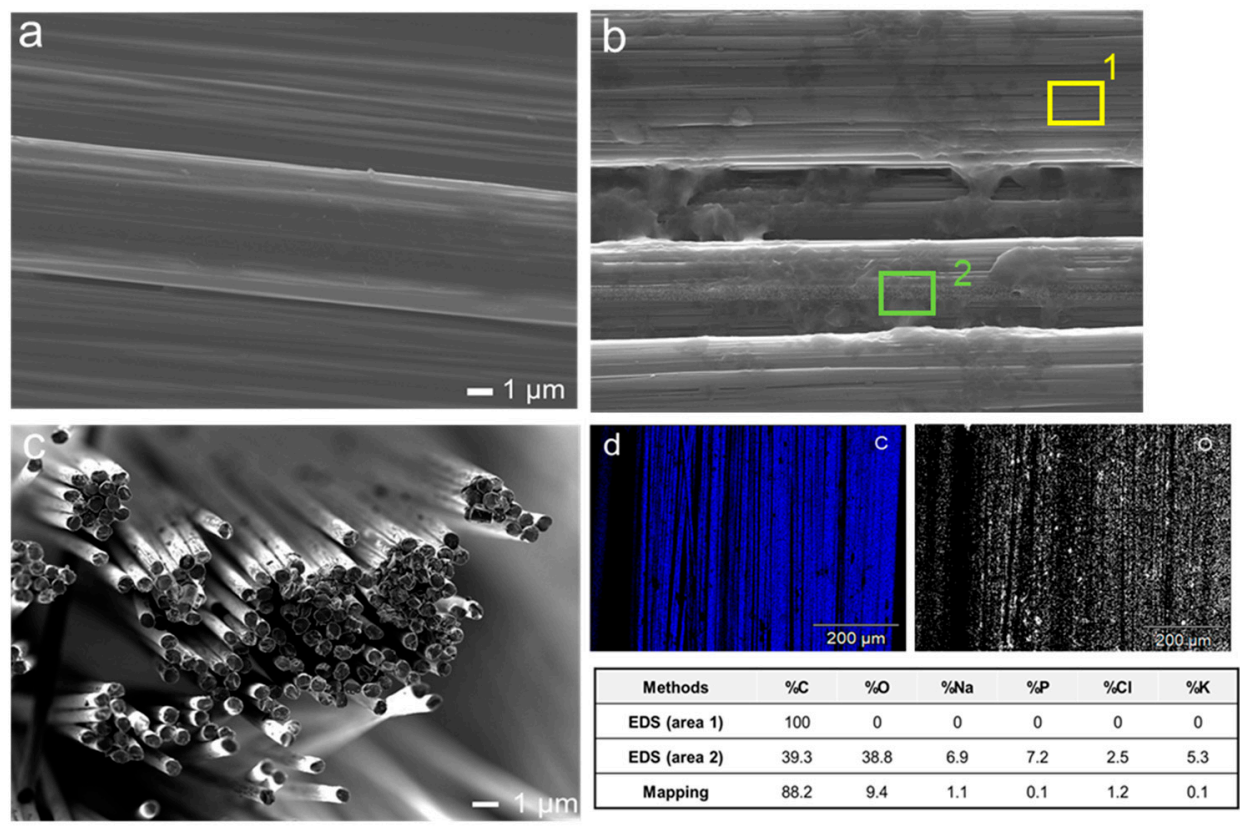

Figure 3. Scanning electron microscope image (a) uncoated carbon fibre; (b) carbon fibre coated with rGO using $3 \mathrm{CV}$ cycles (table gives values for areas not coated (area 1) and coated with rGO (area 2); (c) bundle of bare carbon fibre; (d) Energy-dispersive X-ray spectroscopy (EDS) - mapping images of the $3 \mathrm{CV}$ cycles rGO showing chemical composition of Carbon and Oxygen.

\subsection{Surface Area of the Fibre Electrode}

The electrochemical surface area of the fibre electrodes was calculated using the Randles-Sevcik equation, and cyclic voltammetry at different scan rates in $10 \mathrm{mM}$ $\mathrm{K}_{3} \mathrm{Fe}(\mathrm{CN})_{6}$ containing $0.1 \mathrm{M} \mathrm{KCl}$ as supporting electrolyte. Table 1 shows how the carbon fibre coated from $5 \mathrm{CV}$ cycles gave a 2-fold increase in electrochemical surface area compared to the uncoated carbon fibre, whereas the potentiostatic coating led to only a slight increase in the electrochemical surface area of the fibre electrode.

Table 1. Surface area of uncoated carbon fibre and rGO coated carbon fibre.

\begin{tabular}{lcccc}
\hline & Uncoated Carbon Fibre & Potentiostatic & CV 3 & CV 5 \\
\hline Area of electrode $\left(\mathbf{c m}^{2}\right)$ & 0.09 & 0.11 & 0.19 & 0.20 \\
\hline
\end{tabular}

\subsection{Drug Sensing}

Figure 4a shows the sensing ability of rGO coated on a carbon fibre electrode using 5 $\mathrm{CV}$ cycles, in various concentrations of paracetamol $(0-500 \mu \mathrm{M})$ at a scan rate of $50 \mathrm{mVs}^{-1}$. Paracetamol was found to have an oxidation peak at $0.62 \mathrm{~V}$ (vs. $\mathrm{Ag} / \mathrm{AgCl}$ ) and a reduction peak at $-0.086 \mathrm{~V}$ (vs. $\mathrm{Ag} / \mathrm{AgCl}$ ). This oxidation peak potential is similar to that previously observed with rGO coated on a glassy carbon electrode at $0.63 \mathrm{~V}$ (vs. $\mathrm{Ag} / \mathrm{AgCl}$ ) [1]. A calibration curve for paracetamol sensing measured with this rGO-coated carbon fibre is shown in Figure $4 \mathrm{~b}$, where the peak current was plotted versus concentration. The limit of detection of this rGO-coated fibre sensor was found to be $36.3 \mu \mathrm{M}$ with a linear range 
of $50-500 \mu \mathrm{M}$ of paracetamol $\left(\mathrm{R}^{2}=0.9901\right)$. In control experiments, the oxidation of paracetamol was not evidenced at the uncoated carbon fibre electrode.
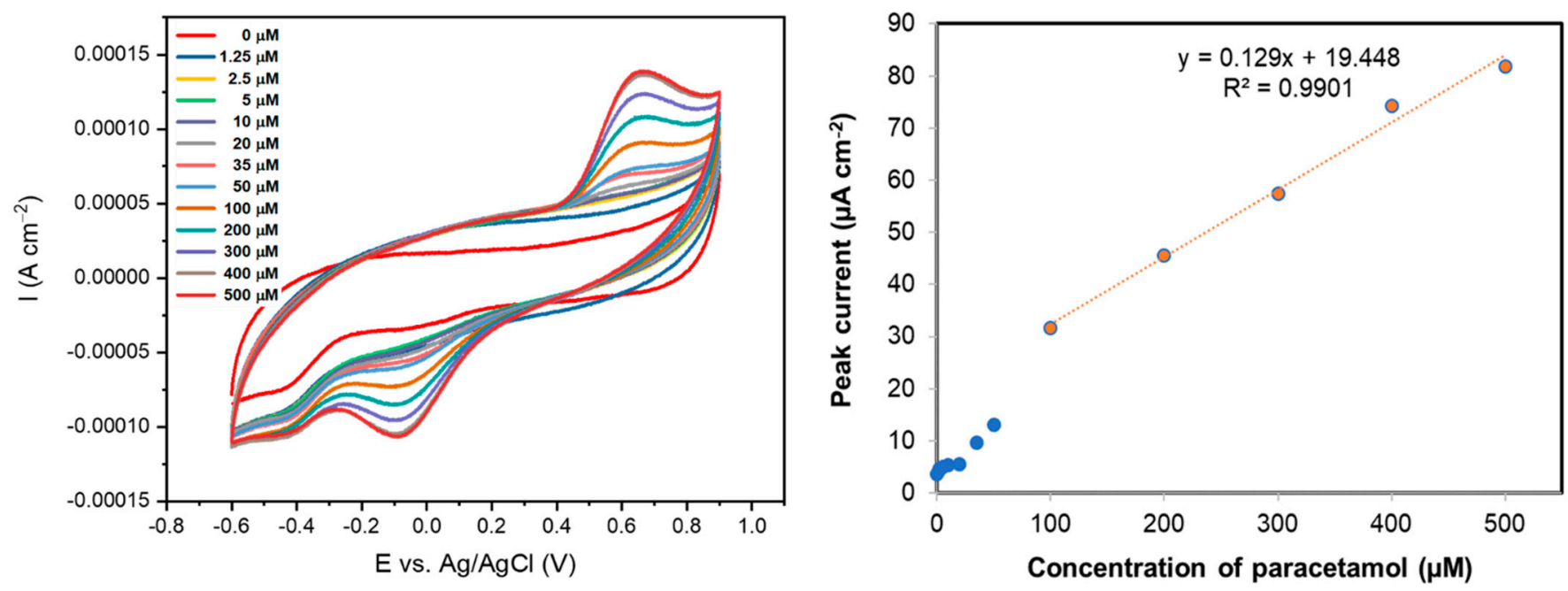

Figure 4. (left) Cyclic voltammograms $\left(50 \mathrm{mV} \mathrm{s}^{-1}\right)$ of paracetamol $(0-500 \mu \mathrm{M})$ in $0.1 \mathrm{M}$ PBS solution using a carbon fibre coated with rGO deposited using $5 \mathrm{CV}$ cycles; (right) Calibration curve for the same rGO-coated carbon fibre in solutions of paracetamol $(0-500 \mu \mathrm{M})$ in $0.1 \mathrm{M}$ PBS solution.

\section{Conclusions}

The electrodeposition of rGO on a carbon fibre electrode via potentiostatic deposition and cyclic voltammetry improved the sensor's ability to detect paracetamol. The electrochemical signal of the rGO-coated fibre deposited using five CV cycles increased with the concentration of paracetamol, resulting in an enhanced sensitivity of the sensor to paracetamol. Further work is underway to sense a broader range of drug molecules and to improve the fibre sensor response with further surface modification.

Acknowledgments: The authors thank the Royal Thai Government Scholarship for providing funding for this work as well as EPSRCs grant, EP/M022749/1.

Conflicts of Interest: The authors declare no conflict of interest and the funders had no role in the design of the study; in the collection, analyses, or interpretation of data; in the writing of the manuscript, or in the decision to publish the results.

\section{References}

1. Ghanbari, K.; Bonyadi, S. An electrochemical sensor based on reduced graphene oxide decorated with polypyrrole nanofibers and zinc oxide-copper oxide p-n junction heterostructures for the simultaneous voltammetric determination of ascorbic acid, dopamine, paracetamol, and tryptoph. New J. Chem. 2018, 42, 8512-8523.

2. Yang, W.; Chen, Y.; Wang, J.; Peng, T.; Xu, J.; Yang, B.; Tang, K. Reduced Graphene Oxide/Carbon Nanotube Composites as Electrochemical Energy Storage Electrode Applications. Nanoscale Res Lett. 2018, 13, 181.

3. Zhang, W.; Shen, C.; Lu, G.; Ni, Y.; Lu, C.; Xu, Z. Synthesis of PPy/RGO-based hierarchical material with super-paramagnetic behavior and understanding its robust photo current driven by visible light. Synth Met. 2018, 241, 17-25.

4. Ponnaiah, S.K.; Prakash, P.; Vellaichamy, B. A new analytical device incorporating a nitrogen doped lanthanum metal oxide with reduced graphene oxide sheets for paracetamol sensing. Ultrason Sonochem. 2018, 44, $196-203$.

5. Jiang, L.; Gu, S.; Ding, Y.; Jiang, F.; Zhang, Z. Facile and novel electrochemical preparation of a graphene-transition metal oxide nanocomposite for ultrasensitive electrochemical sensing of acetaminophen and phenacetin. Nanoscale 2014, 6, 207-214. 